\title{
Full-Squat as a Determinant of Performance in CrossFit
}

\author{
Authors \\ Rafael Martínez-Gómez ${ }^{1,}$, Pedro L. Valenzuela2, 3, ${ }^{*}$, David Barranco-Gil1, Susana Moral-González, \\ Adela García-González ${ }^{4}$, Alejandro Lucia1, 5
}

\section{Affiliations}

1 Universidad Europea de Madrid, Faculty of Sports Science, Madrid, Spain

2 Department of Systems Biology, University of Alcalá, Madrid, Spain

3 Department of Sport and Health, Spanish Agency for Health Protection in Sport (AEPSAD), Madrid, Spain

4 Universidad Pontificia Comillas, School of Nursing and Physiotherapy San Juan de Dios, Madrid, Spain

5 Research Institute Hospital 12 de Octubre, Madrid, Spain

Key words

assessment, resistance exercise, strength, power

accepted $\quad 06.06 .2019$

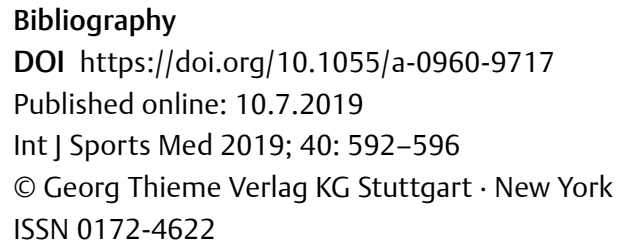

\section{Correspondence}

Mr. Pedro L. Valenzuela, BSc, MRes

Universidad de Alcala de Henares Facultad de Medicina y

Ciencias de la Salud, Systems Biology,

Ctra. Barcelona, Km 33,600

28871 Alcala de Henares

Spain

Tel.: + 34/918/854 522, Fax: + 34/918/854 522

pedro.valenzuela92@gmail.com

\begin{abstract}
This study analyzed the relationship between CrossFit performance and power and strength variables measured in the fullsquat exercise. Twenty male trained subjects ( $33 \pm 7$ years) performed an incremental load full-squat test for assessment of the 1-repetition maximum (1RM) and the mean (Pmean) and peak (Ppeak) power. Performance in 5 different Workouts of the Day (WODs) was measured on different days, and overall CrossFit performance was determined as the sum of the scores obtained in these WODs. Athletes were then assigned to a high (HP) or low (LP) performance group based on the median score for overall performance. Correlation analysis between squat variables and performance was performed and between-group differences were assessed. Moderate to strong $(r=0.47-0.69$, $\mathrm{p}<0.05$ ) positive correlations were found between squat variables and performance in the different WODs. Overall CrossFit performance was strongly and positively associated with absolute $(r=0.62, p=0.01)$ and relative $1 R M(r=0.65, p=0.07)$, and relative Pmean $(r=0.56, p=0.02)$ and Ppeak $(r=0.53, p=0.03)$. Large differences (effect sizes ranging 1.1-1.7, all $p<0.05$ ) were observed between HP and LP for absolute and relative $1 \mathrm{RM}$, relative Pmean, and absolute and relative Ppeak. In summary, strength and power indexes measured in a squat test are positively associated with CrossFit performance.
\end{abstract}

\section{Introduction}

The popularity of CrossFit, a strength and conditioning exercise program, has increased considerably in recent years, with more than 10000 gyms worldwide and over 200000 athletes participating in the annual CrossFit Games [1]. This training modality comprises repeated circuits or series of different gymnastics (e. g., handstands), weight lifting (e. g., clean and jerk), and aerobic exercises (e. g., running), which are combined and performed as

contributed equally quickly as possible within different types of workout sessions known as "Workouts of the Day" (WODs).

There is scarce evidence about the determinants of CrossFit performance, as reported in a recent systematic review [1]. Bellar et al. found that maximal aerobic capacity and peak anaerobic power were related to CrossFit performance, although only in one of the two WODs that were analyzed [2]. Other authors found that overall maximal strength (as determined by the sum of the onerepetition maximum [1RM] in lower and upper-limb resistance exercises) and aerobic capacity were related to performance in two 
out of three WODs [3]. In this respect, analyzing the potential determinants of sport performance might be important for talent identification and sports success [4].

Strength and power indexes recorded in the squat exercise have been related to performance in a variety of sport actions - notably jumps, accelerations, and different weight lifting exercises [5-9]. Given this, and considering that WODs include most of the aforementioned types of actions, we hypothesized that the squat exercise could be used as a predictor of CrossFit performance, at least for exercises involving lower-limb muscles. Correspondingly, higher strength and power index values in this simple test might be related to a greater ability to perform explosive movements such as those included in CrossFit and, accordingly, its routine implementation might provide useful information to predict successful performance in this sport. In light of these considerations, the present study aimed to analyze the relationship between CrossFit performance and power and strength variables measured in the squat exercise.

\section{Materials and Methods}

\section{Participants}

Twenty male subjects participated in this study (age, [mean \pm SD] $33 \pm 7$ years; height, $177 \pm 6 \mathrm{~cm}$; mass, $79 \pm 9 \mathrm{~kg}$ ). Inclusion criteria included being healthy and performing at least three CrossFit sessions per week during the previous year. The protocol was approved by the Institutional Review Board of the Universidad Pontificia de Comillas (Madrid, Spain) and meets the ethical standards of the journal [10]. All of the participants provided written informed consent to participate in this study. They were instructed to maintain their normal dietary pattern and to refrain from doing exercise and consuming ergogenic aids or stimulants (e. g., caffeine) during the study.

\section{Experimental design}

The study lasted six days. Participants performed a squat test on the first day, followed by 5 different WODs (performed in random order) during the next 5 consecutive days. A rest period of $24 \mathrm{~h}$ was provided between two consecutive WODs. We determined performance in each WOD as well as CrossFit overall performance, and the differences between the best and worst performances were also assessed.

\section{Squat test}

Participants performed an incremental free-weight (i. e., not performed with a guided machine) full-squat test. Bar velocity was measured with a linear position transducer (Chronojump; Boscosystem, Barcelona, Spain). The initial load was $20 \mathrm{~kg}$ (i. e., just the bar). Thereafter, the load was increased by $15 \mathrm{~kg}$ (with a three-minute rest between loads) until a constant decrease in mean power was observed. Athletes performed 3 consecutive repetitions with each load and the best result was entered into the analyses. We recorded the mean (Pmean) and peak (Ppeak) concentric power, both expressed in $W$ and $W \cdot \mathrm{kg}^{-1}$. The technique was assessed by a researcher who determined which repetitions were valid. Participants received real-time velocity feedback and were verbally encouraged to make a maximum effort.

The 1 RM was calculated based on the individual load-velocity relationship through linear interpolation, assuming a mean velocity of $0.30 \mathrm{~m} \cdot \mathrm{s}^{-1}$ for the $1 \mathrm{RM}$ value [11]. This method has proven to provide an accurate estimate of the actual 1RM even when using just two load-velocity points [12]. We checked that the linear regression accurately fitted the load-velocity data by considering the correlation coefficient $\left(R^{2}=0.95 \pm 0.06\right)$. The $1 R M$ was expressed in both absolute $(\mathrm{kg})$ and relative (\% body mass) values.

\section{Workouts of the day}

The five WODs used in this study, known as '17.1', '17.2', '17.3', '17.4' and '17.5', were performed in the CrossFit games held in 2017 (for specific details see https:/games.crossfit.com/workouts/ open/2017) and are explained below.

In WOD 1 participants had to perform 225 repetitions of dumbbell snatches (50-lb dumbbells) and burpee box jump-overs (24-inch box) in $\leq 20 \mathrm{~min}$, and the time needed to complete the WOD was measured. Only the data from the participants who completed the WOD in due time were included in the analyses. Accordingly, in this WOD a lower performance time indicates a better performance.

In WOD 2 participants had to perform in $\leq 12$ min the maximum possible number of repeated circuits, each consisting of 50 -feet weighted walking lunge (50-lb dumbbells), toes-to-bar, bar muscleups, and power cleans (50-lb dumbbells). Thus, in this WOD a greater number of repetitions indicates a better performance.

In WOD 3 participants had to perform in $\leq 8 \mathrm{~min}$ the maximum possible number of repeated circuits, each including chest-to-bar pull-ups and squat snatches (with weight progressively increasing from 95-265 lb). Thus, in this WOD a greater number of repetitions indicates a better performance.

In WOD 4 participants had to perform in $\leq 13$ min the maximum possible number of repeated circuits, each consisting of deadlifts (225 lb), wall-ball shots (20 lb-ball to a 10 -feet high target), rowing and handstand push-ups. Thus, in this WOD a greater number of repetitions indicates a better performance.

In WOD 5 participants had to perform in $\leq 40$ min 440 repetitions of thrusters $(95 \mathrm{lb}$ ) and double-unders. Only the data from the participants who completed the WOD in less than 40 min were included in the analyses. In this WOD a lower performance time indicates a better performance.

\section{CrossFit performance}

We assessed performance in each WOD based on the performance time (WODs 1 and 5) or number of repetitions (WODs 2-4), with a lower time or greater number of repetitions indicating a better performance, respectively. Moreover, after each WOD participants obtained a score depending on their classification within the group (one point for the first position, two for the second one, and so on). Overall performance was determined as the sum of the scores attained in the five WODs (only for those subjects who completed all of them), with a lower score indicating a better performance. The median overall score was calculated and participants were divided into a high (HP) and low performance (LP) group. 
- Table 1 Relationship between squat variables and performance in the different Workouts of the Day (WODs).

\begin{tabular}{|c|c|c|c|c|c|c|c|c|c|c|}
\hline & \multicolumn{2}{|c|}{ WOD $1(n=16)$} & \multicolumn{2}{|c|}{ WOD $2(n=19)$} & \multicolumn{2}{|c|}{ WOD $3(n=19)$} & \multicolumn{2}{|c|}{ WOD $4(n=18)$} & \multicolumn{2}{|c|}{ WOD $5(n=18)$} \\
\hline & $\mathbf{r}$ & p-value & $\mathbf{r}$ & p-value & $\mathbf{r}$ & p-value & $\mathbf{r}$ & p-value & $\mathbf{r}$ & p-value \\
\hline 1RM (kg) & -0.67 & 0.005 & 0.57 & 0.01 & 0.57 & 0.01 & 0.57 & 0.01 & -0.42 & 0.09 \\
\hline 1RM (\%BM) & -0.50 & 0.05 & 0.56 & 0.01 & 0.47 & 0.04 & 0.58 & 0.01 & -0.55 & 0.02 \\
\hline Pmean (W) & -0.69 & 0.003 & 0.54 & 0.02 & 0.42 & 0.07 & 0.33 & 0.19 & -0.18 & 0.47 \\
\hline Pmean $\left(\mathrm{W} \cdot \mathrm{kg}^{-1}\right)$ & -0.51 & 0.04 & 0.61 & 0.005 & 0.37 & 0.12 & 0.45 & 0.06 & -0.47 & 0.049 \\
\hline Ppeak (W) & -0.41 & 0.12 & 0.55 & 0.02 & 0.47 & 0.04 & 0.51 & 0.03 & -0.18 & 0.47 \\
\hline Ppeak $\left(\mathrm{W} \cdot \mathrm{kg}^{-1}\right)$ & -0.14 & 0.61 & 0.53 & 0.02 & 0.36 & 0.13 & 0.57 & 0.01 & -0.40 & 0.10 \\
\hline
\end{tabular}

WODs 1 and 5 correspond to time (seconds) needed to perform the task, with less time indicating a better performance. WODs 2, 3 and 4 correspond to the maximum number of repetitions performed in the given time, with a greater number of repetitions indicating a better performance.

Abbreviations: 1RM, one-repetition maximum; BM, body mass; Pmean, mean power; Ppeak, peak power. Note: ' $n$ ' refers to the number of participants completing the different WODs.

\section{Statistical analysis}

Data are presented as mean \pm standard deviation (SD). Normal distribution (Shapiro-Wilk test) and homoscedasticity (Levene's test) of the data were checked before any statistical treatment. Simple linear regression was performed to analyze the relationship between squat variables and CrossFit performance, computing Pearson's correlation coefficients $(r)$ and the equations that describe this relationship. r-values of $0.1,0.3,0.5,0.7$ and 0.9 were considered small, moderate, large, very large and extremely large, respectively [13]. We also assessed the proportion of the variance in CrossFit performance that was explained by the squat variables through the computation of the coefficient of determination $\left(R^{2}\right)$. Unpaired t-tests were conducted to assess differences between groups (i.e., HP vs. LP). Effect sizes (ES, Hedges'g) were calculated to determine the magnitude of the differences, with an ES of 0.2, 0.6, 1.2, 2.0 and 4.0 considered small, moderate, large, very large and extremely large, respectively [13]. All analyses were conducted with the software package SPSS 23.0 (IBM, NY, USA) and the level of statistical significance $(\alpha)$ was set at 0.05 .

\section{Results}

From the initial group of 20 subjects who performed the squat test, 16 completed WOD 1 (mean performance $=1065 \pm 143 \mathrm{~s}$ ), $19 \mathrm{WOD}$ 2 (113 \pm 33 repetitions), 19 WOD 3 (71 \pm 30 repetitions), 18 WOD 4 (187 \pm 31 repetitions), and 18 WOD 5 (1060 $\pm 471 \mathrm{~s})$.

Moderate to strong $(r=0.47-0.69)$ positive correlations were found between most squat variables and performance in the different WODs ( $\triangleright$ Table 1). Performance in each of the five WODs was related to at least two squat variables ( $>$ Table 1 ). Overall CrossFit performance could be measured in 16 subjects and was strongly related to absolute $(\mathrm{kg}, \triangleright$ Fig. $1 \mathrm{a})$ and relative $1 \mathrm{RM}(\%$ body mass, > Fig. 1b), as well as to relative $\left(\mathrm{W} \cdot \mathrm{kg}^{1}\right)$ values of Pmean ( $\triangleright$ Fig. 1c) and Ppeak ( $\triangleright$ Fig. 1d). By contrast, no significant relationships were observed between CrossFit performance and absolute values $(W)$ of Pmean $(r=0.39, p=0.14)$ or Ppeak $(r=0.46$, $\mathrm{p}=0.07$ ).

No significant differences were observed for age or anthropometrical variables between the HP and LP groups ( $\vee$ Table 2). Moderate to large differences were observed between the groups for absolute and relative 1RM, relative Pmean, and absolute or relative Ppeak, but no significant differences $(p=0.16)$ were found for absolute Pmean.

\section{Discussion}

The performance determinants of CrossFit remain to be elucidated [1]. This training modality mainly includes 'explosive' powerrelated actions such as Olympic movements (e.g., snatch, clean and jerk), jumps and sprints. Squat performance indexes have been previously related to most of these actions [5-9]. For instance, the $1 \mathrm{RM}$ in the squat is strongly related to greater strength in the snatch or clean and jerk exercise [5]. Moreover, squat concentric power measures are related to performance in explosive actions such as sprinting or jumping, and are better predictors than isometric and isokinetic strength measures [8]. We therefore hypothesized that a relationship between squat and CrossFit performance would be found at least in those WODs including mostly lower-limb exercises. Our results confirm that strength and power indexes measured in a squat test are positively related to CrossFit performance. Indeed, all WODs were related to at least two squat variables despite including upper-limb exercises such as muscle-ups, chest to bar pull-ups or handstand push-ups. However, further research is needed to determine whether upper-limb exercises (e. g., bench press) can also predict CrossFit performance.

To our knowledge, only two studies have previously examined the relationship between physical ability and CrossFit performance. Bellar et al. reported a significant relationship between performance in the Wingate test and CrossFit performance [2], which is in line with our finding that the capacity of lower-limb muscles to generate power can predict CrossFit performance. Muscle strength has also been related to performance in some WODs [3]. In this context, the squat test allows the analysis of both power and strength abilities, and might therefore represent a practical tool for CrossFit athletes' assessment.

Of note, CrossFit WODs are composed of multimodal exercises including not only strength and power-related actions, but also aerobic exercises such as rowing or actions in which a large range of movement is required. This makes CrossFit a complex sport in which different physical abilities (including stamina, flexibility or 

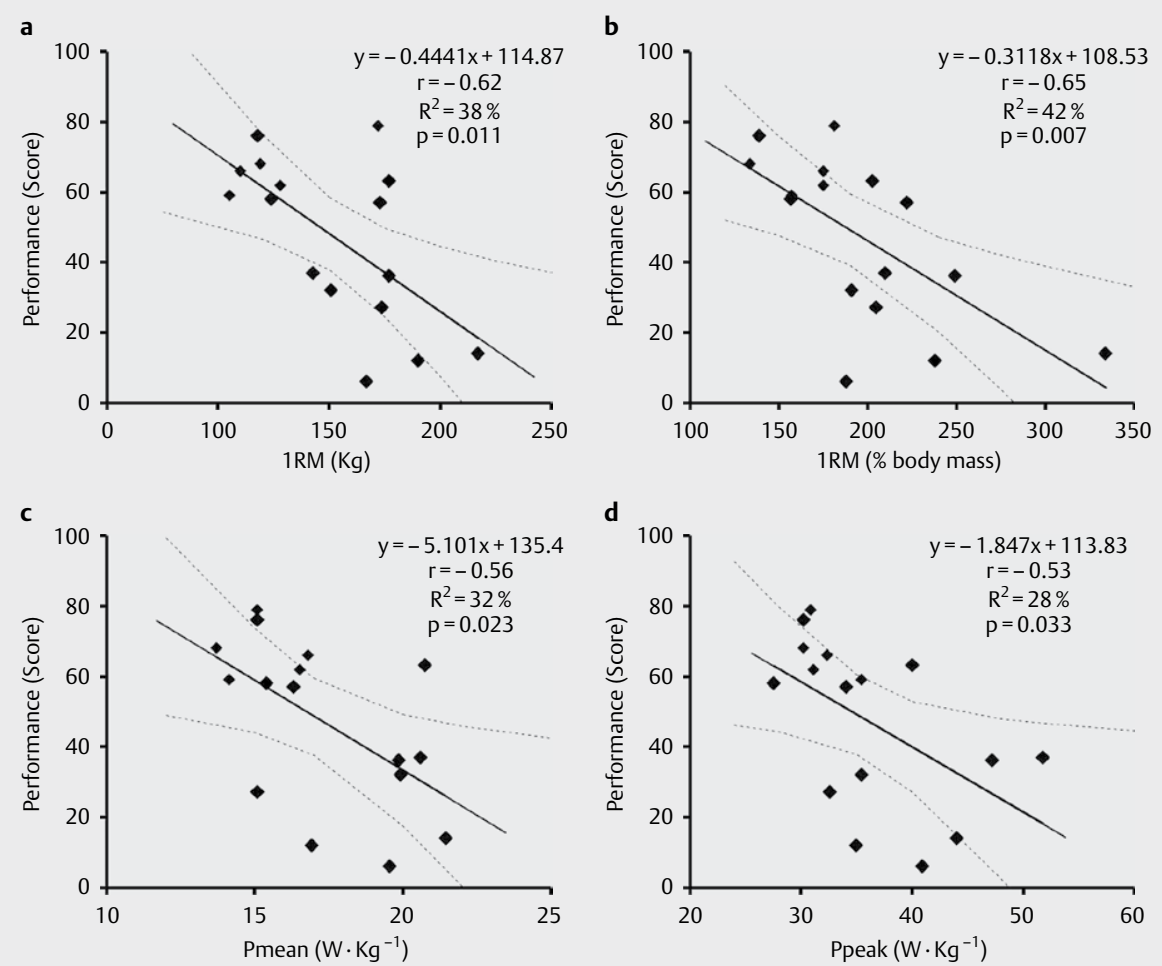

- Fig. 1 Relationship between overall CrossFit performance (i. e., sum of the scores attained in five Workouts of the Day), and absolute ( $\mathrm{kg}$, panel a) and relative values (\% body mass) of 1-repetition maximum (1RM, panel $\mathbf{b}$ ), and relative values $\left(\mathrm{W} \cdot \mathrm{kg}^{-1}\right.$ ) of mean (Pmean, panel $\mathbf{c}$ ) and peak power (Ppeak, panel d). A higher score represents a worse overall result in the five Workouts of the Day performed. Continuous and dashed lines represent line of best fit and $95 \%$ confidence intervals for each correlation, respectively.

- Table 2 Differences between high (HP) and low (LP) performance groups.

\begin{tabular}{|c|c|c|c|c|c|c|}
\hline & $\mathrm{HP}(n=8)$ & $\operatorname{LP}(n=8)$ & p-value & ES & -|trivial|+ & Inference \\
\hline Age (years) & $33 \pm 7$ & $33 \pm 7$ & 0.84 & 0.00 & $26 / 34 / 40$ & Unclear \\
\hline Height $(\mathrm{cm})$ & $177 \pm 7$ & $177 \pm 7$ & 0.83 & 0.00 & $40 / 35 / 25$ & Unclear \\
\hline Body mass $(\mathrm{kg})$ & $77 \pm 8$ & $80 \pm 11$ & 0.57 & 0.29 & $58 / 24 / 18$ & Unclear \\
\hline $\mathrm{BMI}\left(\mathrm{kg} \cdot \mathrm{m}^{-2}\right)$ & $24.6 \pm 1.0$ & $25.3 \pm 2.7$ & 0.50 & 0.33 & $67 / 13 / 19$ & Unclear \\
\hline 1RM (kg) & $174 \pm 23$ & $132 \pm 27$ & 0.005 & 1.58 & $00 / 00 / 100$ & Most likely \\
\hline 1RM (\%BM) & $230 \pm 47$ & $165 \pm 23$ & 0.004 & 1.66 & $00 / 01 / 99$ & Very likely \\
\hline Pmean (W) & $1429 \pm 156$ & $1271 \pm 259$ & 0.161 & 0.70 & $05 / 09 / 86$ & Likely \\
\hline Pmean $\left(\mathrm{W} \cdot \mathrm{kg}^{-1}\right)$ & $18.7 \pm 2.3$ & $15.9 \pm 2.2$ & 0.026 & 1.18 & $01 / 03 / 97$ & Very likely \\
\hline Ppeak (W) & $3051 \pm 387$ & $2567 \pm 470$ & 0.041 & 1.06 & $01 / 03 / 96$ & Very likely \\
\hline Ppeak $\left(\mathrm{W} \cdot \mathrm{kg}^{-1}\right)$ & $40.1 \pm 7.0$ & $32.2 \pm 3.8$ & 0.014 & 1.33 & $00 / 02 / 98$ & Very likely \\
\hline
\end{tabular}

agility) might play a role. For this reason, the squat test should be combined with the assessment of other physical/physiological markers.

In summary, a strong relationship was found between strength and power indexes measured in a squat test, and performance in different WODs (i. e., greater values of strength and power are associated with a lower time to complete the task in WODs 1 and 5 , or an increased number of repetitions in a given time in WODs 2, 3 and 4). Overall CrossFit performance was related to maximal strength and mean and peak power, and these variables appeared as predictors of the best vs. the worst performance levels, respectively, especially when expressed in relative values. Therefore, lower-limb strength (i. e., 1RM) and power (i. e., peak and mean power) indices measured during a squat test can provide valuable information on CrossFit athletic performance, are related to better scores in different WODs (even those including upper-limb exercises), and allow the prediction of overall CrossFit performance. 


\section{Acknowledgements}

The authors gratefully acknowledge Néstor Pérez Mallada and Irene París Zamora (Universidad Pontificia de Comillas, Madrid, Spain) for their help. We also thank all the participants as well as CrossFit Las Rozas (Madrid, Spain) and the head coach Manuel Gómez Martin. The work of Pedro L. Valenzuela is supported by University of Alcalá (FPI2016). The work of Alejandro Lucia is supported by grants from Spanish Ministry of Science, Innovation and Universities and Fondos FEDER (PI15/00558 and PI18/00139).

\section{Conflict of Interest}

The authors declare no conflicts of interest.

\section{References}

[1] Claudino JG, Gabbett TJ, Bourgeois F, HDS Souza, Miranda RC, Mezêncio B, Soncin R, Alberto C, Filho C, Bottaro M, Hernandez AJ, Amadio AC, Serrão JC. CrossFit overview: Systematic review and meta-analysis. Sports Med Open 2018; 4: 11

[2] Bellar D, Hatchett A, Judge LW, Breaux ME, Marcus L. The relationship of aerobic capacity, anaerobic peak power and experience to performance in CrossFit exercise. Biol Sport 2015; 32: 315-320

[3] Butcher S], Neyedly TJ, Horvey KJ, Benko CR. Do physiological measures predict selected crossFit ${ }^{\circledR}$ benchmark performance? Open Access J Sport Med 2015; 6: 241-247

[4] Johnston K, Wattie N, Schorer J, Baker J. Talent identification in sport: A systematic review. Sport Med 2018; 48: 97-109
[5] Carlock JM, Smith SL, Hartman M], Morris RT, Ciroslan DA, Pierce KC, Newton RU, Harman EA, Sands WA, Stone MH. The relationship between vertical jump power estimates and weightlifting ability: A field-test approach. J Strength Cond Res 2004; 18: 534-539

[6] Chelly M, Chérif N, Ben Amar M, Hermassi S, Fathloun M, Bouhlel E, Tabka Z, Shephard R. Relationships of peak leg power, 1 maximal repetition half back squat, and leg muscle volume to 5 -m sprint performance of junior soccer players. J Strength Cond Res 2010; 24: 266-271

[7] Comfort P, Stewart A, Bloom L, Clarkson B. Relationships between strength, sprint, and jump performance in well-trained youth soccer players. J Strength Cond Res 2014; 28: 173-177

[8] Requena B, González-Badillo J, Saez de Villareal E, Ereline J, García I, Gapeyeva H, Pääsuke M. Functional performance, maximal strength, and power characteristics in isometric and dynamic actions lower extremities in soccer players. J Strength Cond Res 2009; 23: 1391-1401

[9] Wisløff U, Castagna C, Helgerud ], Jones R, Hoff J. Strong correlation of maximal squat strength with sprint performance and vertical jump height in elite soccer players. Br J Sports Med 2004; 38: 285-288

[10] Harriss D], Macsween A, Atkinson G. Standards for ethics in sport and exercise science research: 2018 update. Int J Sports Med 2017; 38: 1126-1131, 2009; 23: 1391-1401

[11] Conceição F, Fernandes ], Lewis M, Gonzaléz-Badillo J], Jimenéz-Reyes P. Movement velocity as a measure of exercise intensity in three lower limb exercises. J Sports Sci 2016; 34: 1099-1106

[12] García-Ramos A, Haff GG, Pestaña-Melero FL, Pérez-Castilla A, Rojas F], Balsalobre-Fernández $C$, Jaric $S$. Feasibility of the two-point method for determining the one-repetition maximum in the bench press exercise. Int J Sports Physiol Perform 2018; 13: 478-481

[13] Hopkins W, Marshall SW, Batterham AM, Hanin J. Progressive statistics for studies in sports medicine and exercise science. Med Sci Sports Exerc 2009; 41: 3-12 